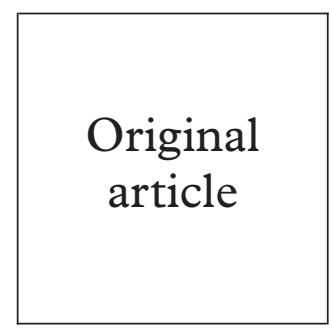

\title{
Asymptomatic non-ulcerative genital tract infections in a rural Ugandan population
}

\author{
L A Paxton, N Sewankambo, R Gray, D Serwadda, D McNairn, C Li, M J Wawer
}

\begin{abstract}
Objective: To document the prevalence of asymptomatic non-ulcerative genital tract infections (GTI) in a rural African cohort.

Methods: The study population consisted of all adults aged 15-59 residing in 56 rural communities of Rakai District, southwest Uganda, enrolled in the Rakai STD Control for AIDS Prevention Study. Participants were interviewed about the occurrence of vaginal or urethral discharge and frequent or painful urination in the previous 6 months. Respondents were asked to provide blood and a first catch urine sample. Serum was tested for HIV-1. Urine was tested with ligase chain reaction (LCR) for $N$ gonorrhoeae and $C$ trachomatis. Women provided two self administered vaginal swabs; one for $T$ vaginalis culture and the other for a Gram stained slide for bacterial vaginosis $(\mathrm{BV})$ diagnosis.
\end{abstract}

Results: A total of 12827 men and women were enrolled. Among 5140 men providing specimens, $0.9 \%$ had gonorrhoea and $2.1 \%$ had chlamydia. Among 6356 women, $1.5 \%$ had gonorrhoea, $2.4 \%$ had chlamydia, $23.8 \%$ were infected with trichomonas and $50.9 \%$ had BV. $53 \%$ of men and $66 \%$ of women with gonorrhoea did not report genital discharge or dysuria at anytime within the previous 6 months. $92 \%$ of men and $76 \%$ of women with chlamydia and over $80 \%$ of women with trichomonas or BV were asymptomatic. The sensitivities of dysuria or urethral discharge for detection of infection with either gonorrhoea or chlamydia among men were only $21.4 \%$ and $9.8 \%$ respectively; similarly, among women the sensitivity of dysuria was $21.0 \%$ while that of vaginal discharge was $11.6 \%$. For trichomonas or BV the sensitivity of dysuria was $11.7 \%$ and that of vaginal discharge was $10.5 \%$.

Conclusion: The prevalence of non-ulcerative GTIs is very high in this rural African population and the majority are asymptomatic. Reliance on reported symptoms alone would have missed $80 \%$ of men and $72 \%$ of women with either gonorrhoea or chlamydia, and over $80 \%$ of women with trichomonas or BV. To achieve STD control in this and similar populations public health programmes must target asymptomatic infections.

(Sex Transm Inf 1998;74:421-425)

Keywords: STDs; gonorrhoea; chlamydia; trichomonas; Uganda

\section{Introduction}

Sexually transmitted diseases (STDs) and other genital tract infections (GTIs) continue to cause significant morbidity and mortality throughout the world. In sub-Saharan Africa, limited economic resources and poor access to medical services have combined to result in STD rates estimated at up to 10 times those found in industrialised nations ${ }^{1}$ and these infections may act as cofactors in HIV transmission. ${ }^{2-4}$ The association between STDs and HIV is strongest for those infections that cause genital ulceration ${ }^{5-7}$ but has also been demonstrated for infections such as gonorrhoea, chlamydia, and Trichomonas vaginalis. ${ }^{8}{ }^{9}$ Recent research suggests that bacterial vaginosis (BV), a common genital tract infection which in pregnant women has been linked to premature labour and low birth weight, may also be associated with HIV risk. ${ }^{10} 11$ Despite the higher risk of HIV acquisition associated with ulcerative diseases, non-ulcerative genital tract infections may theoretically account for a larger proportion of STD associated HIV infection because of their greater prevalence. A corollary to the enhancing effects of STD on HIV acquisition is evidence that HIV infection may in turn exacerbate STD infection and intensify STD transmission. ${ }^{12}$
Most STD control programmes, particularly those based on the syndromic approach, rely on symptom recognition by an infected person who as a consequence seeks medical care. This approach has an inherent weakness in that many infected people either have no symptoms or symptoms so mild as to pass unnoticed. Individuals may also fail to recognise symptoms as manifestations of disease. In general, estimates of the relative magnitude of asymptomatic infections vary by disease and study population. Estimates of the proportion of women with gonorrhoea who are asymptomatic range from $25 \%$ among women attending acute care facilities to $80 \%$ of those referred to STD clinics as sexual partners of infected men. ${ }^{13}{ }^{14} \mathrm{~A}$ retrospective study of men seen at an inner city genitourinary clinic in the United Kingdom found that $10.2 \%$ of those with gonorrhoea had no symptoms. ${ }^{15}$ It is believed that asymptomatic chlamydial infections are even more common with estimated rates among infected men ranging from $50 \%$ to $90 \%$ and more than $80 \%$ among infected women. ${ }^{16-18}$ Estimates of asymptomatic Trichomonas vaginalis among women at STD clinics have ranged from $10 \%$ to $40 \%{ }^{19}$ Bacterial vaginosis is thought to be the most prevalent cause of vaginal symptoms among women of childbearing 
$\operatorname{age}^{20}$ yet, in one study $47 \%$ of women with BV attending an STD clinic were asymptomatic. ${ }^{21}$

Findings from industrialised countries may not be applicable to the developing world. Studies of disease prevalence in Africa have been conducted primarily among people attending STD and antenatal clinics or commercial sex workers and their clients. Chlamydia or gonorrhoea were detected in $6.5 \%$ of pregnant women in Zaire ${ }^{22}$ while rural Tanzanian women attending antenatal clinics also had high rates of asymptomatic infection. ${ }^{23}$ It is not known to what degree prevalence rates among clinic attenders are representative of those in the general population. Partially as a result of the AIDS epidemic, more emphasis has been placed in recent years on the community prevalence of STDs. A recent study which used a combination of symptom assessment and screening for urinary leucocytes to select individuals for further evaluation documented a gonorrhoea and chlamydia prevalence of $2.2 \%$ and $0.7 \%$, respectively, among rural Tanzanian men, the majority of whom had no symptoms. ${ }^{24}$

The Rakai Project has implemented a community based intervention trial in rural Uganda to determine the effect of intensive STD control, via mass treatment, on HIV transmission. As part of the preliminary phase of the study, an extensive door to door survey was conducted among all consenting adults in the study communities. Trained survey personnel collected interview data and biological specimens. In this paper we documented the prevalence of non-ulcerative GTI in the enrolled population and determined the association between laboratory evidence of infection and self reported symptoms of discharge and dysuria.

\section{Methods}

The eligible study population consisted of all adults aged 15-59 years residing in 56 rural communities of Rakai District, in southwest Uganda. After providing written informed consent, participants were interviewed in their homes. Information was collected about their knowledge of STDs and HIV and about measures taken to avoid transmission. Respondents were asked about the occurrence of STD symptoms, including vaginal or urethral discharge and frequent or painful urination, at any time within the preceding 6 months. Both men and women were then asked to provide venous blood and a first catch urine sample. Women were given two cotton Dacron swabs and instructed to insert them, one at a time, deeply into the vagina and gently rotate them for approximately 10 seconds. The interviewer used one swab to inoculate an InPouch TV trichomonas culture media kit (BioMed Diagnostics, San Jose, CA, USA) and rolled the other swab onto a microscope slide. The InPouch TV was incubated at $37^{\circ} \mathrm{C}$ then microscopically examined at 12,24, and 48 hours to detect Trichomonas vaginalis. The InPouch TV test is over $99 \%$ sensitive for the diagnosis of Trichomonas vaginalis. ${ }^{25} 26$ The slide was air dried, Gram stained, and microscopically examined. Diagnosis of bacterial vaginosis
(BV) was determined according to a morphological scoring system ${ }^{27}$ based on the proportions of lactobacilli relative to Gram negative anaerobes. Scores of 7-10 are classified as BV, based on reduced proportion or absence of lactobacilli and a predominance of Gram negative organisms (Gardnerella, Bacteroides, and Mobiluncus morphotypes). Urine samples were separated into $1 \mathrm{ml}$ aliquots. Processed urine specimens were tested for Chlamydia trachomatis and Neisseria gonorrhoeae using ligase chain reaction (LCR) techniques (Abbott Laboratories, Abbott Park, IL, USA) at Johns Hopkins University. Urine based LCR testing for Chlamydia trachomatis and $N$ gonorrhoeae has been shown to have sensitivity and specificity comparable with or higher than culture in both symptomatic and asymptomatic men and women. ${ }^{28-31}$

\section{Results}

The baseline survey for the STD Control for AIDS Prevention Study was begun in November 1994 and completed in July 1995. From a de facto population of 13704 adults aged 15-59 resident for more than 6 months in the study communities and present at the time of survey, a total of $12827(93.6 \%)$ consented to interview. Compliance with specimen provision was high: $90.6 \%$ of those interviewed gave a blood sample, $94.4 \%$ gave urine, and $93.6 \%$ of women provided vaginal swabs. The prevalence rates reported here for trichomonas are based on the total enrolled population providing samples. As LCR examinations are currently expensive, we randomly selected a 30\% subsample of urine specimens from both sexes for testing. Owing to the time consuming nature of microsopic examination of BV slides the data we here report on $\mathrm{BV}$ are based on the $80 \%$ of slides that have been read to date.

The majority of the study population belonged to the Baganda ethnic group. Agriculture and minor commerce were the predominant occupations reported by both men and women. For three quarters of participants the highest level of completed education was primary school, yet almost all respondents (99.7\%) knew about AIDS and over 90\% were able to name several STDs. The majority were sexually active: $76.8 \%$ of females and $78.1 \%$ of males reported engaging in sexual intercourse within the previous 12 months. Almost two thirds of respondents were either married or in a stable union. While over $95 \%$ of married people reported that their most recent sexual encounter was with their spouse, $8.7 \%$ of married men and $1.2 \%$ of married women reported having extramarital relations within the past year. Overall, $28.7 \%$ of men reported two or more sexual partners in the previous year compared with only $3.8 \%$ of women. Condom use was low with only $26.9 \%$ of men and $11.7 \%$ of women reported having ever used them. Although formal commercial sex work is rare in this predominantly rural population, $15.6 \%$ of females and $28.4 \%$ of males acknowledged giving or receiving money or gifts in exchange for sexual intercourse at least once in their lifetime. 
Table 1 Prevalence of non-ulcerative genital tract infection in males and females

\begin{tabular}{|c|c|c|c|c|}
\hline \multirow[b]{2}{*}{ Infection } & \multicolumn{2}{|l|}{ Males } & \multicolumn{2}{|l|}{ Females } \\
\hline & $N$ & $(\%)$ & $N$ & $(\%)$ \\
\hline Gonorrhoea* & $15 / 1716$ & $(0.9)$ & $32 / 2211$ & $(1.5)$ \\
\hline Chlamydia* & $36 / 1716$ & $(2.1)$ & $54 / 2211$ & $(2.4)$ \\
\hline Bacterial vaginosis & NA & NA & $2462 / 4837$ & $(50.9)$ \\
\hline Trichomonas & NA & NA & $1472 / 6183$ & $(23.8)$ \\
\hline
\end{tabular}

$\star$ Rates determined from a subsample. $\mathrm{NA}=$ not applicable.

Table 2 Symptoms associated with gonorrhoea and chlamydia infection in males and females $(n(\%))$

\begin{tabular}{llllll}
\hline Infection & No & $\begin{array}{l}\text { Discharge } \\
\text { alone }\end{array}$ & $\begin{array}{l}\text { Dysuria } \\
\text { alone }\end{array}$ & $\begin{array}{l}\text { Discharge } \\
\text { and dysuria }\end{array}$ & Asymptomatic \\
\hline Males: & 1716 & $1(6.7)$ & $4(26.7)$ & $2(13.3)$ & $8(53.3)$ \\
$\quad$ Gonorrhoea & 15 & 0 & $1(2.8)$ & $2(5.5)$ & $33(91.7)$ \\
$\quad$ Chlamydia & 36 & 0 & $117(7.0)$ & $32(1.9)$ & $1492(89.6)$ \\
$\quad$ Neither infection & 1665 & $24(1.5)$ & & & \\
Females: & 2211 & & & & \\
$\quad$ Gonorrhoea & 32 & $4(12.5)$ & $7(21.9)$ & 0 & $21(65.6)$ \\
$\quad$ Chlamydia & 50 & $2(4.0)$ & $6(12.0)$ & $4(8.0)$ & $38(76.0)$ \\
Both infections & 4 & 0 & $1(25.0)$ & 0 & $3(75.0)$ \\
$\quad$ Neither infection & 2125 & $128(6.0)$ & $172(8.1)$ & $53(2.5)$ & $1772(83.4)$ \\
All subjects: & 3927 & & & & \\
$\quad$ Gonorrhoea & 47 & $5(10.6)$ & $11(23.4)$ & $2(4.3)$ & $29(61.7)$ \\
$\quad$ Chlamydia & 86 & $2(2.3)$ & $7(9.3)$ & $6(7.0)$ & $71(82.6)$ \\
Both infections & 4 & 0 & $1(25.0)$ & 0 & $3(75.0)$ \\
Neither infection & 3790 & $152(4.0)$ & $289(7.6)$ & $85(2.2)$ & $3264(86.1)$ \\
\hline
\end{tabular}

Table 3 Symptoms associated with infection in females tested for both trichomonas and bacterial vaginosis $(B V)(n=5135)(n(\%))$

\begin{tabular}{llllll}
\hline Infection & $\begin{array}{l}\text { No of } \\
\text { subjects }\end{array}$ & $\begin{array}{l}\text { Discharge } \\
\text { alone }\end{array}$ & $\begin{array}{l}\text { Dysuria } \\
\text { alone }\end{array}$ & $\begin{array}{l}\text { Discharge } \\
\text { and dysuria }\end{array}$ & Asymptomatic \\
\hline BV & 1785 & $128(7.2)$ & $151(8.5)$ & $57(3.2)$ & $1449(81.1)$ \\
Trichomonas & 801 & $48(6.0)$ & $87(10.9)$ & $24(3.0)$ & $642(80.1)$ \\
Both & 654 & $58(8.9)$ & $36(5.5)$ & $25(3.8)$ & $535(81.8)$ \\
Neither & 1895 & $124(6.5)$ & $151(8.0)$ & $57(3.0)$ & $1563(82.5)$ \\
\hline
\end{tabular}

Overall prevalence of GTIs was high and was higher among females than males (table 1) The frequency of symptoms among subjects with LCR proved gonorrhoea or chlamydia is shown in table 2. The proportion of asymptomatic infections was very high: $63 \%$ of the 51 study subjects infected with gonorrhoea and $82 \%$ of the 90 subjects with chlamydia reported no symptoms within the 6 months before interview. For both sexes, those with gonorrhoea tended to be more symptomatic than those with chlamydia. As shown in table 3, $81 \%$ of 2439 women with bacterial vaginosis and $80 \%$ of 1455 women with trichomonas were asymptomatic.

There were sex and infection specific differences in symptom reporting among those infected with gonorrhoea or chlamydia. A greater proportion of men with chlamydia $(91.7 \%)$ were without symptoms of discharge or dysuria than women $(76.0 \%)$. However, asymptomatic infection with gonorrhoea was less frequent in males than females $(53.5 \% v$ $65.6 \%$ ) (table 2) Among men, the sensitivity of dysuria as a means of identifying gonorrhoea or chlamydia was low for both infections but was roughly four times greater for those with gonorrhoea than for those with chlamydia: $40.0 \%$ for $N$ gonorrhoeae compared with only $8.3 \%$ for $C$ trachomatis, while the sensitivity of urethral discharge for $N$ gonorrhoeae was $20.0 \%$ versus $5.6 \%$ for $C$ trachomatis. The sensitivity of dysuria and vaginal discharge was also very low among women but, in contrast with men, there was little difference between women with chlamydia and those with gonorrhoea. Both for women with gonorrhoea and women with chlamydia, the sensitivity of dysuria was $22 \%$ to $24 \%$ while the sensitivity of vaginal discharge was only $11 \%$ (table 4 ). Most algorithms for the syndromic diagnosis and treatment of STDs used in the developing world call for treatment of both $C$ trachomatis and $N$ gonorrhoeae when a patient has symptoms of dysuria and/or genital discharge. We therefore calculated the sensitivities of those symptoms for infection with either organism and found the sensitivity of dysuria and discharge among males in this population to be only $21.4 \%$ and $9.8 \%$. Among females, the respective figures were $20.9 \%$ and $11.6 \%$. In both sexes the positive predictive value of either dysuria or discharge for detection of either gonorrhoea or chlamydia was no higher than $8.2 \%$.

Because symptoms and treatment of bacterial vaginosis and Trichomonas vaginalis are similar, we considered the two infections together. Sixty three per cent of women had either trichomonas or bacterial vaginosis on the day of interview, yet among 5135 women for whom we had information on both infections, only $10.5 \%$ reported vaginal discharge and $11.7 \%$ reported dysuria any time within the previous 6 months, neither of which was statistically different from women who had no evidence of vaginal infection (table 3) However, because of the high prevalence of these infections the positive predictive value of dysuria and vaginal discharge was relatively high $(65 \%)$.

Table 4 Sensitivity, specificity, and positive predictive value of symptoms associated with genital tract infection in males and females

\begin{tabular}{|c|c|c|c|c|c|c|}
\hline \multirow[b]{2}{*}{ Infection } & \multicolumn{3}{|l|}{ Discharge } & \multicolumn{3}{|l|}{ Dysuria } \\
\hline & $\begin{array}{l}\text { Sensitivity } \\
(\%)\end{array}$ & $\begin{array}{l}\text { Specificity } \\
(\%)\end{array}$ & $\begin{array}{l}P P V \\
(\%)\end{array}$ & $\begin{array}{l}\text { Sensitivity } \\
(\%)\end{array}$ & $\begin{array}{l}\text { Specificity } \\
(\%)\end{array}$ & $\begin{array}{l}P P V \\
(\%)\end{array}$ \\
\hline \multicolumn{7}{|l|}{ Males: } \\
\hline Gonorrhoea & 20.0 & 96.6 & 4.9 & 40.0 & 91.1 & 3.8 \\
\hline Chlamydia & 5.6 & 96.5 & 3.3 & 8.3 & 90.8 & 1.9 \\
\hline Gonorrhoea or chlamydia & 9.8 & 96.6 & 8.2 & 21.4 & 91.1 & 5.7 \\
\hline \multicolumn{7}{|l|}{ Females: } \\
\hline Gonorrhoea & 11.1 & 91.4 & 2.1 & 22.2 & 89.2 & 3.3 \\
\hline Chlamydia & 11.1 & 91.4 & 3.1 & 24.0 & 89.2 & 5.3 \\
\hline Gonorrhoea or chlamydia & 11.6 & 91.5 & 5.2 & 20.9 & 89.4 & 7.4 \\
\hline Bacterial vaginosis & 11.0 & 90.6 & 51.4 & 11.0 & 88.2 & 45.7 \\
\hline Trichomonas & 10.7 & 90.1 & 29.8 & 11.8 & 88.7 & 29.3 \\
\hline $\begin{array}{l}\text { Bacterial vaginosis or } \\
\text { trichomonas }\end{array}$ & 10.5 & 90.4 & 65.3 & 11.7 & 89.0 & 64.6 \\
\hline
\end{tabular}


Respondents were asked about the occurrence of dyspareunia and pelvic pain, but on analysis neither of these additional symptoms was found to augment the sensitivity of discharge or dysuria for any of the infections studied.

\section{Discussion}

The cornerstone of STD control is treatment of infected people both to cure the index case and to prevent further disease transmission. In the developing world, laboratory resources are scarce and, therefore, for the most part, treatment is based on syndromic diagnosis. Previous clinic based evaluations of the efficacy of syndromic treatment algorithms have shown that the sensitivity of symptoms of discharge and/or dysuria to determine infection with non-ulcerative GTI is poor. ${ }^{22}{ }^{23}$ This study has shown that their utility is even lower among the general population. The ability of LCR to detect $C$ trachomatis and $N$ gonorrhoeae in urine samples and the use of self administered vaginal swabs for the diagnosis of $T$ vaginalis and bacterial vaginosis obviated the need for genital and pelvic examinations and thus allowed collection of biological specimens in the subjects' homes. We achieved high rates of participation and, because entire communities were enrolled, the study cohort was representative of the population at large and not limited by self selection of those who attend clinics. This high compliance and avoidance of selective participation coupled with highly sensitive and specific diagnostic techniques enabled us to document community infection prevalence rates and to compare these with population based symptom reporting.

We found that reliance on reported symptoms alone to detect infection in this population would have missed $80 \%$ of men and $72 \%$ of women with either gonorrhoea or chlamydia, and over $80 \%$ of women with trichomonas or BV. This was a point prevalence survey and we recognise the possibility of overrepresentation of asymptomatic infections as people with symptoms are more likely to seek treatment and thus not be detected by the survey. Data from this cohort reveals that $40 \%$ of people with symptoms do not seek treatment at all and that of those who do, only two thirds go to locations such as government or private clinics and health centres where they might receive effective therapy. It is also possible that some of the infected people in this survey were in an early, presymptomatic stage. However, because the diagnostic techniques used identify infections at all stages of disease the majority of infected people were presumably at stages where they might be expected to have already experienced symptoms. In this study we matched laboratory evidence of infection to symptoms occurring at any time in the previous 6 months. If we had restricted symptom reporting to a shorter period around the time of sample collection, the proportion reporting symptoms would have been even lower and the proportion of asymptomatic infections correspondingly higher. In addition, the low rates of reported symptoms occurred despite detailed interview. Presumably, the proportion of infected people who would have self identified and thus sought treatment would have been even lower without probing by the interviewer.

There were people who reported symptoms yet had no evidence of infection and it is probable that many of these symptoms were caused by disease processes other than those investigated. Alternatively, some people may have been infected and symptomatic with one of the GTIs of interest during the 6 month symptom recall period, yet because they were no longer infected on the day of interview, we were unable to confirm that the reported symptom had been associated with infection. For those with infections diagnosed at the time of interview, maximising the reporting period increased the likelihood of eliciting a symptom and thereby increased the apparent sensitivity of that symptom. However, the unavoidable inclusion of an unknown proportion of people who may in the past have been both infected and symptomatic may have decreased the apparent specificity and thus the positive predictive value of that symptom.

There are several possible explanations for the low symptom reporting among infected people in this population. Biological factors such as the strain of infecting organisms or the infection load may influence the severity of symptoms. ${ }^{32}$ Infection load may in turn be related to the duration of infection and, in settings where infection is highly endemic, one would expect a higher proportion of prevalent as opposed to incident infection. If prevalent infections are indeed associated with fewer symptoms then populations such as this one would tend to have more asymptomatic infections than those with lower disease prevalence. Alternatively, social or cultural factors may lead to the perception of symptoms of infection as "normal". ${ }^{33}{ }^{34}$ Finally, infections may be genuinely and frequently asymptomatic. The practical implication is that if an infected person does not have symptoms or perceives these symptoms as unimportant such a person is unlikely to seek treatment or to reduce his or her sexual activity. Thus, the persistence of unrecognised infection makes it difficult to interrupt transmission and to reduce exposure in a population.

The population represented by this cohort is typical of those found in many parts of rural sub-Saharan Africa. After more than a decade of intensive health education campaigns most respondents are knowledgeable about AIDS and STDs yet use of condoms to prevent infection is low and access to medical services poor. Although few people report engaging in high risk activities such as commercial sex, most adults are sexually active and many have concurrent or overlapping sexual partnerships. This combination of factors has promoted and maintained high levels of infection. Untreated asymptomatic infections have been linked to numerous sequelae including pelvic inflammatory disease, infertility, and potentially to HIV transmission. This latter possibility in particular is of great public health importance. In Tanzania, a community based trial of improved 
STD syndromic treatment that targeted asymptomatic infections in only a very limited manner via screening for urinary leucocytes nevertheless demonstrated a $38 \%$ reduction in $\mathrm{HIV}$ incidence in the intervention arm. This occurred despite minimal STD effects. ${ }^{35}$ It has been suggested that asymptomatic infections may not be as strong a cofactor in HIV transmission, presumably because of less inflammation and lowered HIV viral shedding. It is not known whether or to what extent the transmission likelihood of asymptomatic STD infections differs from that of symptomatic ones and their relative importance in disease deserves further investigation. However, because asymptomatic infections constitute the vast majority of infections in this cohort, it is possible that they may be responsible for much STD transmission and may thus be a substantial barrier to STD control. To have the greatest impact on transmission we must move beyond reliance on syndromic treatment alone and investigate more novel approaches that also target asymptomatic infections.

Individual contributions from authors not available.

1 Meheus A, Schultz K, W Cates. Development of prevention and control programs for sexually transmitted diseases in developing countries. In: K Holmes, P Mardh, eds. Sexuall transmitted diseases. 2nd ed. New York: McGraw-Hill, 1990:1041

2 Quinn T. Association of sexually transmitted diseases and infection with the human immunodeficiency virus: biologif STD AIDS 1996;7(Suppl 2):17-24.

3 Wasserheit JN. Epidemiological synergy: interrelationships between human immunodeficiency virus infection and other sexually transmitted diseases. Sex Transm Dis 1992;19:61-77

4 Clottey C, Dallabetta G. Sexually transmitted diseases and human immunodeficiency virus. Epidemiologic synergy? human immunodeficiency virus.

5 Wasserheit. J. The significance and scope of reproductive tract infections among third world women. Int $\mathcal{F}$ Gynecol Obstet 1989;(Suppl 3): 145-68.

6 Plummer FA, Simonsen JN, Cameron DW, et al. Cofactors in male-female sexual transmission of human immunodeficiency virus type 1. F Infect Dis 1991;163:233-9.

7 Plourde PJ, Pepin J, Agoki E, et al. Human immunodeficiency virus type 1 seroconversion in women with genita ulcers. F Infect Dis 1994;1 170:313-7.

8 Weir SW, Feldblum PJ, Roddy RE, et al Gonorrhea as a risk factor for HIV acquisition. AIDS 1994;8:1605-8.

9 Laga M, Manoka A, Kivuvu M, et al. Non-ulcerative sexually transmitted diseases as risk factors for HIV-1 transmission in women: results from a cohort study. AIDS 1993;7: 95-102.

10 Sewankambo N, Gray RH, Wawer MJ, et al. Human immunodeficiency virus type 1 infection associated with
abnormal vaginal flora morphology and bacterial vaginosis. abnormal vaginal flora m

11 Cohen CR, Duerr A, Pruithithada N, et al. Bacterial vaginosis and HIV seroprevalence among female commercial sex workers in Chiang Mai, Thailand. AIDS 1995;9:1093-7.

12 Wald A, Corey L, Handsfield $\mathrm{H}$, et al. Influence of HIV infection on manifestations and natural history of other sexually transmitted disease. Annu Rev Publ Health 1993;14:19-42.
13 Pedersen AHB, Bonin P. Screening females for asymptomatic gonorrhea infection. Northwest Med 1971;70:255.

14 McCormack WM, Stumacher RJ, Johnson K, et al. Clinica spectrum of gonococcal infection in women. Lancet 1977;2:1182-5

15 Sherrard J, Barlow D. Gonorrhoea in men: clinical and diagnostic aspects. Genitourin Med 1996;72:422-6.

16 Joshi JV, Palayekar S, Hazari KT, et al. The prevalence of Chlamydia trachomatis in young women. Natl Med F India 1994;7:57-9.

17 Sessa R, Latino MA, Magliano EM, et al. Epidemiology of of urogenital infections caused by Chlamydia trachomatis and outline of characteristic features of patients at risk. $\mathcal{F}$ Med Microbiol 1994;41:168-72.

18 Stamm W, Holmes K. Chlamydia trachomatis infections of the adult. In: K Holmes, P Mardh, et al, eds. Sexually transmitted diseases. 2nd ed. New York: McGraw-Hill, 1990: 181-5.

19 Rein M, Muller M. Trichomonas vaginalis and trichomoniasis. In: K Holmes, P Mardh, et al, eds. Sexually transmit ted diseases. 2nd ed. New York: McGraw-Hill, 1990:484.

20 Hillier S, Holmes K. Bacterial vaginosis. In: K Holmes, P Mardh, et al, eds. Sexually transmitted diseases. 2nd ed. New Mardh, et al, eds. Sexually transn

21 Eschenbach DA, Hillier S, Critchlow C, et al. Diagnosis and clinical manifestations of bacterial vaginosis. Am $\mathcal{F}$ Obstet Gynecol 1988;158:819.

22 Vuylsteke B, Laga M, Alary M, et al. Clinical algorithms for the screening of women for gonococcal and chlamydial infection: evaluation of pregnant women and prostitutes in Zaire. Clin Infect Dis 1993;17:82-8.

23 Mayaud P, Grosskurth H, Changalucha J, et al. Risk assessment and other screening options for gonorrhoea and ment and other screening options for gonorrhoea and chlinics. Bull World Health Organ 1995;73:621-30.

24 Grosskurth H, Mayaud P, Mosha F, et al. Asymptomatic gonorrhoea and chlamydial infection in rural Tanzanian men. BMF 1996;312:277-280.

25 Borchardt KA, Smith RF. An evaluation of an InPouch TV culture method for diagnosing Trichomonas vaginalis infection. Genitourin Med 1991;67:149-52.

26 Draper D, Parker R, Patterson E, et al. Detection of Trichomonas vaginalis in pregnant women with the InPouch TV Culture System. F Clin Microbiol 1993;31: 1016-18.

27 Nugent RP, Krohn MA, Hillier SL. Reliability of diagnosing bacterial vaginosis is improved by a standard method of gram stain interpretation. f Clin Microbiol 1991;29:297301 .

28 Smith K, Ching S, Lee H, et al. Evaluation of ligase chain reaction for use with urine for identification of Neisseria gonorrhoeae in females attending a sexually transmitted disease clinic. 7 Clin Microbiol 1995;33:455-7.

29 Dille B, Butzen C, Birkenmeyer L. Amplication of Chlamydia trachomatis DNA by ligase chain reaction. I Clin Microbiol 1993;31:729-31.

30 Buimer M, Van Doornum GJ, Ching, et al. Detection of Chlamydia trachomatis and Neisseria gonorrhoeae by ligase chain reaction-based assays with clinical specimens ligase chain reaction-based assays with clinical specimens
from various sites: implications for diagnostic testing and from various sites: implications for diagnostic
screening. $\mathcal{F}$ Clin Microbiol 1996;34:2395-400.

31 Chernesky MA, Lee H, Schacter J, et al. Diagnosis of Chlamydia trachomatis urethral infection in symptomatic and asymptomatic men by testing first-void urine in a ligase chain reaction assay. F Infect Dis 1994;170:1308-11.

32 Ross JD, Wardropper A, Sprott M, et al. Gonococcal infection in Edinburgh and Newcastle: serovar prevalence in relation to clinical features and sexual orientation. Genitourin Med 1994;70:35-9.

33 Green EC. Sexually transmitted disease, ethnomedicine and health policy in Africa. Soc Sci Med 1992;35:121-30.

34 Mulder D. Disease perception and health-seeking behavior for sexually transmitted diseases. In: Prevention and management of sexually transmitted diseases in Eastern and management of sexually transmitted diseases in Eastern and Southern Africa: current approaches and future directions. NARESA Mon
Nairobi, 1994 .

35 Grosskurth H, Mosha F, Todd J, et al. Impact of improved treatment of sexually transmitted diseases on HIV infection in rural Tanzania: randomised control trial. Lancet 1995;346:530-6. 